\title{
High-level persistence of biofilm formation in in methicillin resistant Staphylococcus aureus
}

\author{
Manjunath Chavadi ${ }^{1}$, Rahul Narasanna ${ }^{1}$, Ramu Manjula ${ }^{2}$ and Chandrakanth R. Kelmani ${ }^{1 *}$ \\ ${ }^{1}$ Department of P.G. Studies and Research in Biotechnology Gulbarga University, Gulbarga, Karnataka, India. \\ ${ }^{2}$ National Institute of Mental Health and Neurosciences, Department of Biophysics, Bangalore, Karnataka, \\ India. \\ Email: ckelmani@gmail.com
}

Received 13 February 2018; Received in revised form 2 August 2018; Accepted 9 August 2018

\begin{abstract}
Aims: Biofilm formation by Methicillin-resistant Staphylococcus aureus on a variety of surfaces and detection of the biofilm-forming population by the most reliable method is very much essential to diagnose the nosocomial infection caused by $S$. aureus.

Methodology and results: This study is aimed to evaluate the biofilm producing ability of $S$. aureus by qualitative Congo red agar (CRA), and quantitative microtitre plate (MTP) methods. The morphological difference of biofilms analysis was done by SEM (Scanning Electron Microscope) and genotyping analysis of mecA and femA for determination of MRSA among isolated $S$. aureus strains and to check the biofilm producers among MRSA strains. Biofilm production was found to be at different intensities by MTP. The strong, moderate and weak biofilm producers were found to be $38.63 \%, 31.81 \%$, and $29.54 \%$ respectively. The strong adherent biofilm formed by representative isolate developed a dense biofilm with thick mucus three-dimensional multilayered structure of macroscopic dimension. Conversely, SEM analysis of moderate and weak biofilm representative strain failed to form a monolayer of scattered single cells to three-dimensional structure. The $47.72 \%$ of $S$. aureus isolates have shown positive for the genotypic analysis of mecA and femA. The strong and moderate biofilm forming MRSA was found to be $38.63 \%$ and $9.09 \%$, respectively.

Conclusion, significance and impact of study: The great challenge is associated with biofilm mediated infection caused $S$. aureus healthy and hospitalized individual hence the present study reinforces the need of precautionary measures to avoid the indiscriminate use of antibiotics in case of biofilm-forming MRSA.
\end{abstract}

Keywords: MRSA, MSSA, Biofilm, MTP and SEM.

\section{INTRODUCTION}

Health-care-associated infections caused by microorganisms pose a significant problem in a present day hospital environment, among various microbial infections, Staphylococcus aureus infection is one of the most frequently occurring bacterial infections (Smith et al., 2008). The continuous persistence of $S$. aureus believed to be because of its ability to resist antibiotics. For instance, the antibiotic methicillin was being used against $S$. aureus, however, within two years $S$. aureus found resistant to methicillin (Eriksen, 1961). The emergence and widespread of Methicillin resistant $S$. aureus MRSA between the early 1960 s and late 1990s have begun to pose a serious threat to the chemotherapy of Staphylococcal diseases; this resistivity led to the difficulty in successful treatment of $S$. aureus infections (Eguia and Chambers, 2003). The genetic determinant of methicillin resistance in MRSA is the acquired gene $m e c A$, which encodes the low-affinity penicillin-binding protein $2 \mathrm{~A}$ (PBP2A) and the current theory, this functions as a surrogate transpeptidase in the presence of high concentration of $\beta$ lactam antibiotics that inactivate four high-affinity PBPs native to $S$. aureus (de Jonge and Tomasz, 1993). In India alone, $70 \%$ of the $S$. aureus strains are resistant to methicillin which has increased mortality rate (Anupurba et al., 2003). Compared to other pathogenic strains (MRSA) infections caused $20 \%$ death of all infections (Boucher and Corey, 2008). The resistivity of $S$. aureus infections is dependent on several factors among them, the most important factor is the ability of this organism to form biofilms (Babra et al., 2014).

Several multidrug-resistant bacteria including $S$. aureus are capable of forming a monolayer of scattered single cells to thick multi-layered mucus like the structure of the macroscopic dimension known as a biofilm on the various surface (Mirani et al., 2013). Biofilm formed by multidrug-resistant MRSA protect the cells not only from 
host immune response but also from antimicrobial agents by restricted penetration of antibiotic through the biofilm, nutrient limitation, adaptive response and genetic alteration to the prevailing cell (Mirani and Jamil, 2011). Biofilm persistence is the cause of many chronic infections such as endocarditis, osteomyelitis, chronic otitis media, foreign-body-associated infections, gastrointestinal ulcers and urinary tract infections (Anupurba et al., 2003). Apart from infection, biofilm helps bacteria to communicate with each other by using chemical signal called quorum sensing, the cell-cell communication via quorum sensing involves the production, release, detection, and response to small hormone-like molecules termed pheromones or autoinducers (Jayaraman and Wood, 2008). The mechanism of biofilm formation includes the Staphylococci attachment either to host tissue or to the surface of a medical device, they then proliferate and accumulate into multi-layered cell clusters, which are embedded in an amorphous extracellular material that mainly is composed of $\mathrm{N}$-acetyl-glucosamine, cell wall teichoic acids, DNA, and host products (Heilmann and Götz, 2010). The mature biofilm contains fluid-filled channels that ensure the delivery of nutrients and oxygen to bacterial cells located deeper in the biofilm. From a mature biofilm, individual cells or cell aggregates can detach, and upon detachment, the bacteria may disseminate via the bloodstream, which may cause infection (Anupurba et al., 2003; Heilmann and Götz, 2010).

It becomes important to check for the presence of biofilms in a particular area in order to control them. In this regard, the present study is an attempt to detect biofilm producing $S$. aureus in the sample isolated from the hospitals and diagnostic centers of Gulbarga region, Karnataka using antibiotic susceptibility test. We also tested for mecA and femA gene in the isolated $S$. aureus established the difference in the microscopic characters between biofilm producing and non-producing strains.

\section{MATERIALS AND METHODS}

\section{Bacterial strains and growth conditions}

In this study, S. aureus will be isolated from cultured pus and blood samples collected from hospitals and diagnostic clinical centers of Gulbarga region, Karnataka, India. Al the samples were enriched using brain heart infusion (BHI) agar (Himedia Laboratories, Mumbai, India) for $24 \mathrm{~h}$ at $37{ }^{\circ} \mathrm{C}$. Isolation and identification of $S$. aureus were monitored on differential and selective media (Baird Parker Agar, Mannitol Salt Agar, and Sheep's blood Agar). Further, isolates were identified on the basis of microscopic morphology, and biochemical studies.

\section{Antibiotic susceptibility test}

Antibiotics susceptibility was determined by disc diffusion method, according to the criteria of the Clinical and Laboratory Standards Institute guidelines (CLSI, 2012). The antibiotic susceptibility of $S$. aureus test was determined using different antibiotics discs namely, penicillin, ampicillin, gentamycin, streptomycin, kanamycin, teicoplanin, methicillin and vancomycin performed on Muller Hinton Agar (Hi-media, Mumbai). The plates were incubated at $37^{\circ} \mathrm{C}$ for $24 \mathrm{~h}$. The strains were classified as resistant, intermediate and susceptible based on the zone of inhibition. Bacterial strains that demonstrated resistance to three or more categories of antibiotics were defined as multidrug resistant.

\section{Phenotypic detection of biofilm producers}

Phenotypic evaluation of biofilm producers was done using the congo red agar as described earlier with slight modification (Freeman et al. 1989; Mirani and Jamil 2011). Brain heart infusion agar plates containing $50 \mathrm{~g} / \mathrm{L}$ sucrose and $0.8 \mathrm{~g} / \mathrm{L}$ Congo red were prepared and streaked with isolates and incubated for $48-72 \mathrm{~h}$ at $37{ }^{\circ} \mathrm{C}$. Positive isolates were indicated by black colonies.

\section{Biofilm assay by Microtitre Plate (MTP)}

The quantitative method of Biofilm forming ability was measured by adherence to microtitre plates (MTP) (Nasr et al., 2014; Christensen et al., 1985). The individual wells of flat-bottom 96-well tissue culture plates (Nunc, Denmark) were filled with $100 \mu \mathrm{L}$ of Tryptic Soy Broth (TSB). Diluted overnight bacterial culture (1:100 in TSB, $200 \mu \mathrm{L}$ of TSB (with/without supplements) was added to each individual well. Well, containing only TSB was used as negative control and incubated for $48 \mathrm{~h}$ at $37^{\circ} \mathrm{C}$. After incubation wells were gently washed with $200 \mu \mathrm{L}$ of PBS $(\mathrm{pH} 7.4)$ then dried in an inverted position in a hot air oven at $60{ }^{\circ} \mathrm{C}$ for $1 \mathrm{~h}$. The wells were stained with $100 \mu \mathrm{L}$ of $2 \%$ crystal violet solution and incubated for $15-20 \mathrm{~min}$ at 37 ${ }^{\circ} \mathrm{C}$. The plates were washed two times with distilled water and dried, then washed using 95\% ethanol, and the optical density was determined using a microtiter plate reader at $570 \mathrm{~nm}$ (iMark Microplate Reader SigmaAldrich, Japan). Based on OD the isolate was referred to as weak $(O D=0.480)$, moderate $(O D=0.720)$ and strong (OD>0.720) biofilm producers (Babra et al. 2014).

\section{Scanning Electron Microscope (SEM) analysis}

Three biofilm forming $S$. aureus isolates (referred to as weakly adherent, moderate adherent, and full adherent strains of $S$. aureus) were based on their MTP biofilm assay, the bacterial cells from each culture were recovered by centrifugation at 6000 RPM for $5 \mathrm{~min}$ and the cells were washed twice with potassium phosphate buffer $(50 \mathrm{mM}, \mathrm{pH} 7.0)$. Bacterial cells were fixed by immersing in $2.5 \%$ glutaraldehyde in potassium phosphate buffer $(50 \mathrm{mM}, \mathrm{pH} 7)$ for overnight at $4{ }^{\circ} \mathrm{C}$. Then the specimens were washed twice with buffer and dehydrated by ethanol series (v/v) ranging from 30\%, $40 \%, 50 \%, 60 \%, 70 \%, 80 \%, 90 \%$ to $100 \%$. For SEM analysis, all the specimens were dried overnight in a desiccator to the critical point, coated with gold and subjected to SEM (Hibbing et al., 2010). 


\section{PCR for detection of mecA and femA genes}

The genomic DNA from $S$. aureus strains was isolated by using the DNA extraction kit (Genei Bangalore) according to the manufacturer's instruction. All the isolates were further subjected for molecular characterization to detect the presence of $m e c A$ and femA gene, associated with the low affinity of penicillin-binding protein, PBP2a. The mecA gene was amplified by PCR using the forward primer $5^{\prime}$ ACTGCTATCCACCCTCAAAC-3' and the reverse primer 5'-CTGGTGAAGTTGTAATCTGG-3', and the femA gene was amplified using the forward primer 5' AAAAAAGCACATAACAAGCG-3'and the reverse primer 5'-GATAAAGAAGAAACCAGCAG-3' (Arakere et al., 2005).

\section{RESULTS}

\section{Antibiotic susceptibility test}

A total of 44 isolates were identified as $S$. aureus, from the different clinical samples (Figure 1), based on colonial characters on different media such as black colonies with opaque zone on Baird Parker agar, mannitol fermentation on mannitol salt agar (Himedia) and blood hemolysis on Sheep's blood agar and further they were confirmed by biochemical tests. The antimicrobial susceptibility test of all 44 isolates showed significant resistance pattern where, $100 \%$ isolates showed resistant to penicillin, $86 \%$ to isolates showed resistant to ampicillin, $75 \%$ to streptomycin, $65.9 \%$ to gentamicin, $54.5 \%$ oxacillin, $52.27 \%$ to methicillin, $34.09 \%$ isolates to ciprofloxacin, $22.7 \%$ to rifampicin, $38.6 \%$ to erythromycin, $11.3 \%$ to vancomycin and $9.09 \%$ to teicoplanin. Among the 44 isolates, the majority of them were having multiple drug resistance (Figure 2 and Table 1).

\section{Biofilm formation}

The results of a current study by the qualitative method by Congo red agar and quantitative method by MTP for biofilm production by 44 isolates were mentioned in Table 1. In Congo red agar method, $54.54 \%$ were biofilm producer; the remaining $45.46 \%$ did not show characteristic features for biofilm production. In MTP method biofilm production was detected with different intensities, $38.63 \%$ isolates were placed in the high biofilm producers, while $31.81 \%$ and $29.54 \%$ strains were placed under the moderate and weak or non-biofilm producers respectively. The correlation between CRA and MTP method for the biofilm detection is represented in Figure 3.

\section{Scanning electron microscope analysis}

SEM analysis (Figure 4) results show that in strong adherent biofilm population the cells were closely intact with thick mucus and multi-layered structure. The moderate adherent biofilm population representative isolate was less loosely intact, grew in uniform monolayer, however, did not form multi-layered and three-dimension biofilm. The weak biofilm isolates failed to aggregate to form a monolayer or a mature biofilm structure.

\section{PCR for detection of femA and mecA genes}

All the isolates were subjected for molecular characterization to detect the presence of mecA and femA gene, associated with the low affinity of penicillin-binding protein, PBP2a. The genotypic expression of methicillin resistance for all $S$. aureus clinical isolates were examined in this study, among total strains of $S$. aureus, $47.72 \%$ were MRSA, all the MRSA strains were positive for the mecA and femA genes amplification which is highly conserved in Staphylococcus species, so it is possible to detect methicillin-resistant $S$. aureus by detection of these gene complex in their genome. The remaining $52.26 \%$ is MSSA which is negative for the mecA gene amplification (Table 1 and 2).

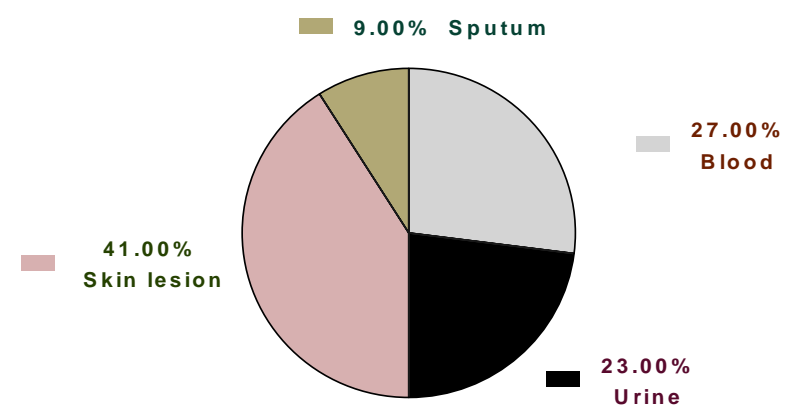

Figure 1: Distribution of $S$. aureus isolates from clinical samples.

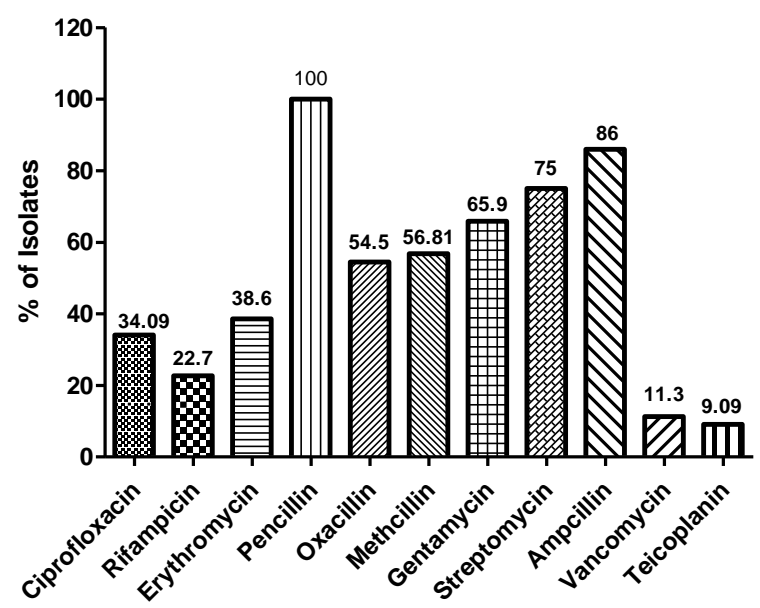

\section{Antibiotics}

Figure 2: Antibiotic profile of $S$. aureus isolates examined in this study. 
Mal. J. Microbiol. Vol 15(1) 2019, pp. 1-7

DOI: http://dx.doi.org/10.21161/mjm.116217

Table 1: Distribution of $m e c A / f e m A$ genes in multidrug-resistant $S$. aureus isolates and evaluation of biofilm production in staphylococcal isolates according to MTP and CRA methods.

\begin{tabular}{|c|c|c|c|c|}
\hline S. aureus strains & $\begin{array}{c}\text { Antibiotic } \\
\text { resistance pattern }\end{array}$ & mecA/femA & CRA & MTP \\
\hline SA1 & POMGSACREVT & + & + & ++ \\
\hline SA2 & PGSA & - & - & + \\
\hline SA3 & POMGSACREVT & + & + & ++ \\
\hline SA4 & PGSA & - & - & $+/-$ \\
\hline SA5 & POMGSACRE & + & + & ++ \\
\hline SA6 & PGA & - & + & $+/-$ \\
\hline SA7 & POMGSACREVT & + & + & ++ \\
\hline SA8 & POMGSACREV & + & + & ++ \\
\hline SA9 & POMGSA & - & + & + \\
\hline SA10 & PSAC & - & - & + \\
\hline SA26 & POMGSA & + & + & + \\
\hline SA12 & POMGSACRE & + & + & ++ \\
\hline SA13 & POMGSA & + & - & + \\
\hline SA14 & PSAC & - & - & $+/-$ \\
\hline SA15 & PGA & - & - & $+/-$ \\
\hline SA16 & POMGSACREVT & + & + & ++ \\
\hline SA17 & PSAE & - & - & + \\
\hline SA18 & POMGSACE & + & + & ++ \\
\hline SA19 & PSA & - & - & + \\
\hline SA20 & POMGSAC & + & + & ++ \\
\hline SA23 & PAE & - & - & + \\
\hline SA25 & POMGSACE & + & + & ++ \\
\hline SA27 & PRE & - & + & $+/-$ \\
\hline SA29 & POMGSAC & + & + & ++ \\
\hline SA30 & POMGSAC & + & - & ++ \\
\hline SA31 & POMGSA & & + & $+/-$ \\
\hline SA32 & POMGSA & & + & $+/-$ \\
\hline SA33 & POMGSACE & + & + & ++ \\
\hline SA34 & PARE & - & - & + \\
\hline SA35 & POMGSA & + & + & + \\
\hline SA36 & POMGSA & + & + & + \\
\hline SA37 & PR & - & - & $+/-$ \\
\hline SA38 & POMGSAE & + & + & ++ \\
\hline SA39 & $P$ & - & + & $+/-$ \\
\hline SA40 & POMGSAE & + & - & ++ \\
\hline SA41 & PAE & - & - & + \\
\hline SA42 & POMGSA & + & + & ++ \\
\hline SA43 & POMGSA & + & + & ++ \\
\hline SA44 & PS & - & - & $+/-$ \\
\hline SA45 & PA & - & - & $+/-$ \\
\hline SA46 & $P$ & - & - & $+/-$ \\
\hline SA47 & PSA & - & - & + \\
\hline SA48 & PMGSA & - & - & + \\
\hline SA49 & $P$ & - & - & $+/-$ \\
\hline
\end{tabular}

$\mathrm{P}$, penicillin; A, ampicillin; S, streptomycin; G, gentamycin; T, tetracycline; C, ciprofloxacin; O, oxacillin; M, methicillin; R, rifampicin; V, vancomycin; E, erythromycin. For typing of mecA and femA, and CRA: $(-)$, negative; $(+)$, positive. For MPT: $(+/-)$, weak; $(+)$, moderate; $(++)$, strongly positive.

\section{DISCUSSION}

In India, the prevalence rate of MRSA is increasing drastically, and in the early 20th century, the prevalence rate of MRSA from India has increased to $20-32.8 \%$ (Udaya Shankar et al., 1997; Mehta et al., 1998). A study from north India, Delhi had also shown the similar results where the rate of MRSA prevalence was $31.2 \%$ and $38.44 \%$ in 2008 and 2009, respectively (Tiwari et al., 2008; Gadepalli et al., 2009). In another study, the rate of MRSA prevalence was found to be $42 \%$ in 2008 and $40 \%$ in 2009 (Ray et al., 2013). According to the previous study, the prevalence rate of MRSA was $44.8 \%$ in 
Gulbarga region (Mendem et al., 2016). However, the prevalence rate of MRSA has been increased now to $54.45 \%$ for the same region.

The MRSA isolated in the present study were resistant to $\geq 10$ antibiotics from a different class of antibiotics including $\beta$-lactamase, aminoglycosides, and glycopeptides. The percentage of MDR isolates were about $72 \%$ which were significantly high in comparison to another study, where it was found to be $20 \%$ (Goldstein et al., 1996). The mechanism for the creation of highly resistant subclones would be the transposition of mobile genetic elements such as integrons, transposons and even larger resistant gene clusters such as SCC mec chromosomal cassettes (Ito et al., 2001; Katayama et al., 2000). Another evidence suggests that the reason for multi-drug resistance may be likely due to prolonged and repeated usage of methicillin against $S$. aureus infection (Martins et al., 2007).

Given the significant negative impact of the nosocomial infection caused by MDR associated biofilm production, the standard test for the detection of biofilm formation is by using MTP. The MTP assay is widely considered to be most sensitive, accurate and reproducible screening method for determination and reported as a reliable quantitative method for detection of different intensities of biofilm formation by the clinical isolates of MDR compare to the qualitative method by congo red agar (Nasr et al., 2012).

In MTP method biofilm production was detected with different intensities, where $38.63 \%$ isolates were placed in the high biofilm producers, while $31.81 \%$ and $29.54 \%$ strains were placed under the moderate and weak biofilm producers respectively. Similar results were observed in the previous study, where $57.8 \%$ of Staphylococcal clinical isolates have displayed positive phenotypic results for biofilm production and $14.47 \%$ were high, and $39.4 \%$ were moderate biofilm producers by MTP method (Mathur et al., 2006). According to another study, the biofilm production was detected in $46 \%$ of the Staphylococcal isolates where, $26 \%$ isolates were strong producers, $12 \%$ isolates were moderate biofilm producers (Nasr et al., 2012).

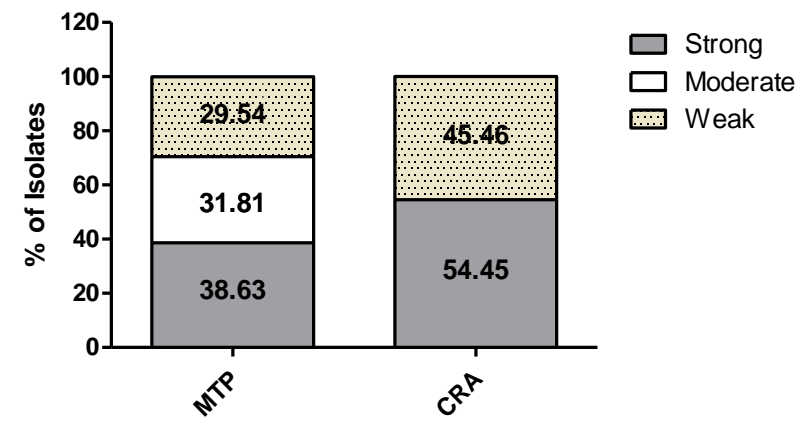

Figure 3: Percentage of biofilm-forming $S$. aureus strains on TSB media according to microtitre plate and Congo red agar method.
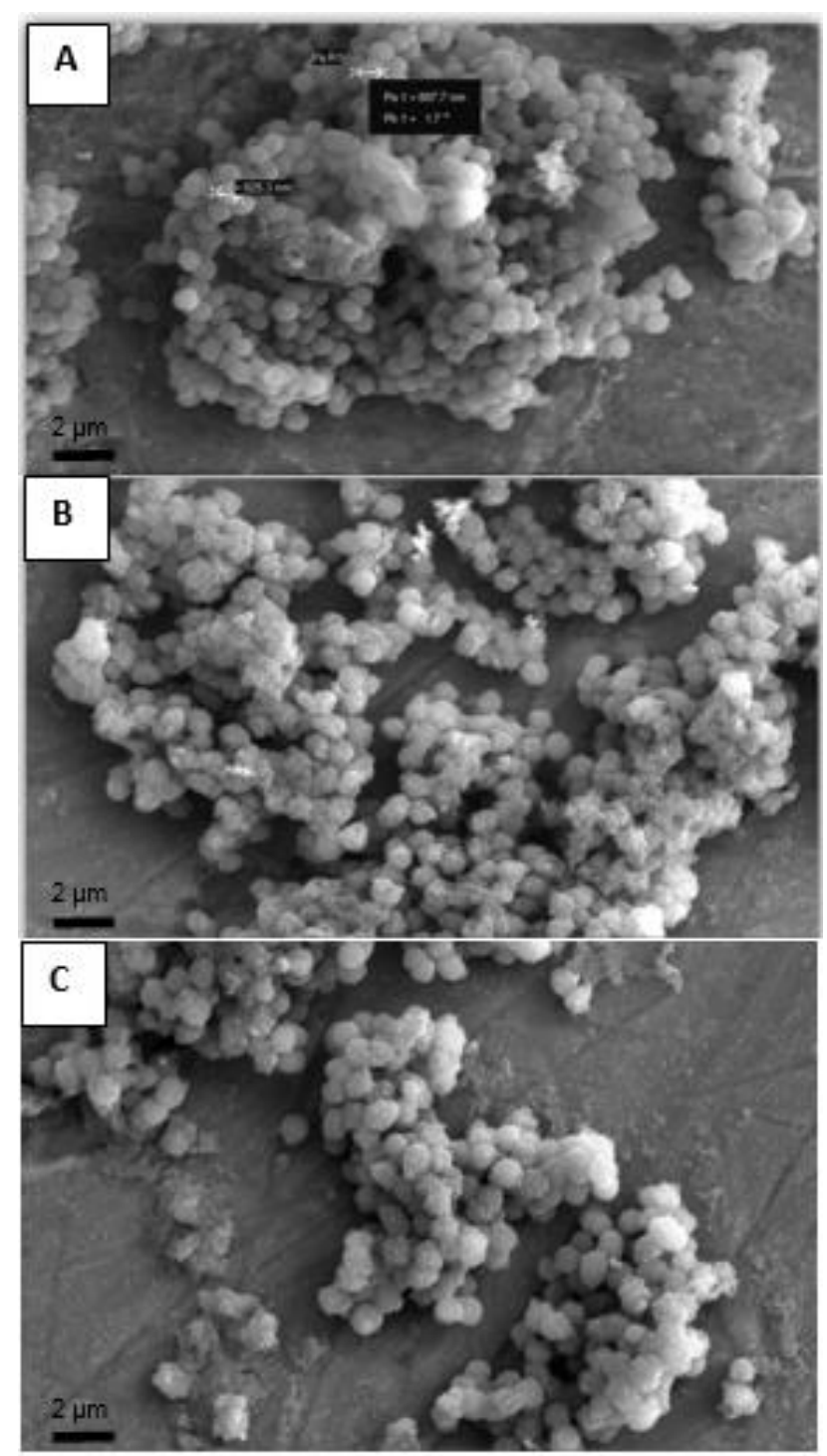

Figure 4: Scanning electron microscope analysis of biofilm formation of $S$. aureus isolates. A, strong adherent biofilm production; B, moderate adherent biofilm production; C, weak or non-adherent biofilm production.

Table 2: Percentage of biofilm-forming $S$. aureus strains on TSB media according to microtitre plate and Congo red agar method. Detection of $m e c A / f e m A$ genes in $S$. aureus isolates.

\begin{tabular}{ll}
\hline Biofilm detection & $(+)$ mecA/femA(\%) \\
\hline MTP & $17(38.63)$ \\
Strong Biofilm & $4(9.09)$ \\
Moderate Biofilm & - \\
Weakly Biofilm & \\
CRA & $18(40.90)$ \\
Biofilm formation &
\end{tabular}

$(-)$, negative; (+), positive, (\%), percentage. 
In the current study, the biofilm production was high in isolates with multidrug-resistant to the different class of antibiotic. This data suggests the significant correlation between prevalence of multidrug-resistant of the clinical isolates and biofilm production. According to the previous study, once $S$. aureus adopts biofilm mode of growth, they protect themselves by resisted penetration of the antibiotic into the cells located in the inner layers of biofilm and the adaptive response to repeated exposure to a different group of antibiotics could induce biofilm formation among the MDR challenging the treatment by antibiotics.

In order to evaluate the monolayer of scattered single cells to thick mucus multi-layered structure of the macroscopic dimension of $S$. aureus morphology, was performed. The changes in morphology noted as an adaptive response to overcome the adverse environmental conditions mainly occurred due to multidrug-resistant in this study and this response has already been reported with several bacterial species (Raju et al., 2007).

The mecA gene amplification from $S$. aureus is considered as a gold standard method for identification of MRSA strains, and it is associated with drug resistance determinant (Arakere et al., 2005; Raju et al., 2007). Further, femA and mecA gene encode for $\beta$-lactam antibiotic resistance and also serves to be a unique feature as a species identification marker of $S$. aureus (Vannuffel et al., 1995; Serralta et al., 2001).

The study suggests that the isolates were MRSA as well as biofilm producers. In this regard, we further extended our work in the determination of biofilm producer among the isolated strains of MRSA and MSSA. The biofilm formation by MTP method is $70.44 \%$ among the total isolates of $S$. aureus which was screened for biofilm formation. The majority of fully and moderate established biofilms were formed by $38.63 \%$ and $9.09 \%$ of MRSA respectively. In contrast, $22.72 \%$ and $29.54 \%$ contributes to moderate adherent and weak biofilm were formed by MSSA. while our study shows $47.72 \%$ of Methicillinresistant strains were biofilm producers compare to methicillin-sensitive strains, which is quite high compared to study showing results that $37.9 \%$ of MRSA strains were biofilm producers (Kwon et al., 2008).

\section{CONCLUSION}

The results suggested that the formation of biofilm helps the representative to resist the antibiotic. The prevalence of biofilm formation was significantly higher in isolates of MRSA compared to MSSA which suggests the dependence of biofilm development on the presence of multidrug resistant. Our results suggest reinforcing the need to look for precautionary and aseptic measures to be taken to prevent the biofilm formation among MRSA and the indiscriminate use of antibiotics at present times in clinical practice.

\section{REFERENCES}

Anupurba, S., Sen, M. R., Nath, G., Sharma, B. M., Gulati, A. K. and Mohapatra, T. M. (2003). Prevalence of methicillin resistant Staphylococcus aureus in a tertiary referral hospital in eastern Uttar Pradesh. Indian Journal of Medical Microbiology 21(1), 49.

Arakere, G., Nadig, S., Swedberg, G., Macaden, R., Amarnath, S. K. and Raghunath, D. (2005). Genotyping of methicillin-resistant Staphylococcus aureus strains from two hospitals in Bangalore, South India. Journal of Clinical Microbiology 43(7), 31983202.

Babra, C., Tiwari, J., Costantino, P., Sunagar, R., Isloor, S., Hegde, N., and Mukkur, T. (2014). Human methicillin-sensitive Staphylococcus aureus biofilms: potential associations with antibiotic resistance persistence and surface polysaccharide antigens. Journal of Basic Microbiology 54(7), 721-728.

Boucher, H. W. and Corey, G. R. (2008). Epidemiology of methicillin-resistant Staphylococcus aureus. Clinical Infectious Diseases 46(Supplement_5), S344-S349.

Christensen, G. D., Simpson, W. A., Younger, J. J., Baddour, L. M., Barrett, F. F., Melton, D. M. and Beachey, E. H. (1985). Adherence of coagulasenegative staphylococci to plastic tissue culture plates: a quantitative model for the adherence of staphylococci to medical devices. Journal of Clinical Microbiology 22(6), 996-1006.

CLSI, (2015). M02-A12 Performance Standards for Antimicrobial Disk Susceptibility Tests. Approved Standard, 12th Edn. Clinical and Laboratory Standards Institute. Wayne, PA. USA.

de Jonge, B. L. and Tomasz, A. (1993). Abnormal peptidoglycan produced in a methicillin-resistant strain of Staphylococcus aureus grown in the presence of methicillin: functional role for penicillin-binding protein $2 \mathrm{~A}$ in cell wall synthesis. Antimicrobial Agents and Chemotherapy 37(2), 342-346.

Eguia, J. M. and Chambers, H. F. (2003). Communityacquired methicillin-resistant Staphylococcus aureus: epidemiology and potential virulence factors. Current Infectious Disease Reports 5(6), 459.

Eriksen, K. R. (1961). "Celbenin"-resistant Staphylococci. Ugeskr Laeger 123, 384-386.

Freeman, D. J., Falkiner, F. R. and Keane, C. T. (1989). New method for detecting slime production by coagulase negative Staphylococci. Journal of Clinical Pathology 42(8), 872-874.

Gadepalli, R., Dhawan, B., Kapil, A., Sreenivas, V., Jais, M., Gaind, R. and Udo, E. E. (2009). Clinical and molecular characteristics of nosocomial meticillinresistant Staphylococcus aureus skin and soft tissue isolates from three Indian hospitals. Journal of Hospital Infection 73(3), 253-263.

Goldstein, E. J., Citron, D. M. and Nesbit, C. A. (1996). Diabetic foot infections: bacteriology and activity of 10 oral antimicrobial agents against bacteria isolated from consecutive cases. Diabetes Care 19(6), 638-641. 
Heilmann, C. and Götz, F. (2010). Cell-cell communication and biofilm formation in gram-positive bacteria. Bacterial Signaling, 1, 7-22.

Hibbing, M. E., Fuqua, C., Parsek, M. R. and Peterson, S. B. (2010). Bacterial competition: surviving and thriving in the microbial jungle. Nature Reviews Microbiology 8(1), 15.

Ito, T., Katayama, Y., Asada, K., Mori, N., Tsutsumimoto, K., Tiensasitorn, C. and Hiramatsu, K. (2001). Structural comparison of three types of staphylococcal cassette chromosome mec integrated in the chromosome in methicillin-resistant Staphylococcus aureus. Antimicrobial Agents and Chemotherapy 45(5), 1323-1336.

Jayaraman, A. and Wood, T. K. (2008). Bacterial quorum sensing: signals, circuits, and implications for biofilms and disease. Annu. Rev. Biomed. Eng. 10, 145-167.

Katayama, Y., Ito, T. and Hiramatsu, K. (2000). A new class of genetic element, staphylococcus cassette chromosome mec, encodes methicillin resistance in Staphylococcus aureus. Antimicrobial Agents and Chemotherapy 44(6), 1549-1555.

Kwon, A. S., Park, G. C., Ryu, S. Y., Lim, D. H., Lim, D. Y., Choi, C. H. and Lim, Y. (2008). Higher biofilm formation in multidrug-resistant clinical isolates of Staphylococcus aureus. International Journal of Antimicrobial Agents 32(1), 68-72.

Martins, A., Couto, I., Aagaard, L., Martins, M., Viveiros, M., Kristiansen, J. E. and Amaral, L. (2007). Prolonged exposure of methicillin-resistant Staphylococcus aureus (MRSA) COL strain to increasing concentrations of oxacillin results in a multidrug-resistant phenotype. International Journal of Antimicrobial Agents 29(3), 302-305.

Mathur, T., Singhal, S., Khan, S., Upadhyay, D. J., Fatma, T. and Rattan, A. (2006). Detection of biofilm formation among the clinical isolates of staphylococci: an evaluation of three different screening methods. Indian Journal of Medical Microbiology 24(1), 25.

Mehta, A. P., Rodrigues, C., Sheth, K., Jani, S., Hakimiyan, A. and Fazalbhoy, N. (1998). Control of methicillin resistant Staphylococcus aureus in a tertiary care centre: A five year study. Indian Journal of Medical Microbiology 16(1), 31.

Mendem, S. K., Gangadhara, T. A., Shivannavar, C. T. and Gaddad, S. M. (2016). Antibiotic resistance patterns of Staphylococcus aureus: A multi center study from India. Microbial Pathogenesis 98, 167-170.

Mirani, Z. A., Aziz, M., Khan, M. N., Lal, I., ul Hassan, N. and Khan, S. I. (2013). Biofilm formation and dispersal of Staphylococcus aureus under the influence of oxacillin. Microbial Pathogenesis 61, 66-72.

Mirani, Z. A. and Jamil, N. (2011). Effect of sub-lethal doses of vancomycin and oxacillin on biofilm formation by vancomycin intermediate resistant Staphylococcus aureus. Journal of Basic Microbiology 51(2), 191-195.

Nasr, R. A., AbuShady, H. M. and Hussein, H. S. (2012). Biofilm formation and presence of icaAD gene in clinical isolates of staphylococci. Egyptian Journal of Medical Human Genetics 13(3), 269-274.

Ray, P., Manchanda, V., Bajaj, J., Chitnis, D. S., Gautam, V., Goswami, P. and Rao, R. (2013). Methicillin resistant Staphylococcus aureus (MRSA) in India: prevalence and susceptibility pattern. The Indian Journal of Medical Research 137(2), 363.

Raju, S., Chandrakanth, R. K. and Patil, S. A. (2007). High-level oxacillin and gentamycin resistance with reduced susceptibility to vancomycin in Staphylococcus aureus-carrying mecA and femA gene complex. Current Microbiology 54(6), 429-434.

Serralta, V. W., Harrison-Balestra, C., Cazzaniga, A. L., Davis, S. C. and Mertz, P. M. (2001). Lifestyles of bacteria in wounds: presence of biofilms? Wounds 13(1), 29-34.

Smith, K., Perez, A., Ramage, G., Lappin, D., Gemmell, C. G. and Lang, S. (2008). Biofilm formation by Scottish clinical isolates of Staphylococcus aureus. Journal of Medical Microbiology 57(8), 10181023.

Tiwari, H. K., Sapkota, D. and Sen, M. R. (2008). High prevalence of multidrug-resistant MRSA in a tertiary care hospital of northern India. Infection and Drug Resistance 1, 57.

Shankar, C. U., Harish, B. N., Kumar, P. U., and Navaneeth, B. V. (1997). Prevalence of methicillin resistant Staphylococcus aureus in JIPMER Hospital-a preliminary report. Indian Journal of Medical Microbiology 15(3), 137.

Vannuffel, P., Gigi, J., Ezzedine, H., Vandercam, B., Delmée, M., Wauters, G. and Gala, J. L. (1995). Specific detection of methicillin-resistant Staphylococcus species by multiplex PCR. Journal of Clinical Microbiology 33(11), 2864-2867. 